\title{
Moving towards life-long User Modeling
}

\author{
Demetris Kyriacou Hugh C. Davis \\ Learning Societies Lab \\ School of Electronics and Computer Science \\ University of Southampton \\ $\{d k e 06 r, h c d\} @ e c s . s o t o n . a c . u k$
}

\begin{abstract}
User Modeling is the core component for the majority of personalization services. It has become an important factor in the effort to personalise a diversity of web resources according to users' characteristics and needs. While trying to move a step further and model dynamic users in a range of contexts, life-long User Modeling, the ability to model a dynamic and changing user throughout lifetime interactions with a variety of resource providers, appears to be an attractive solution. A Scrutable User Modeling Infrastructure is proposed in this paper, where a first prototype has already been designed and implemented, which blends together some key User Modeling 'ingredients'- interoperability, scrutability, user privacy - and attempts to model the various life-long user interactions with a diversity of web-based systems from the educational, business and social networking domains.
\end{abstract}

\section{Introduction}

What if each one of us had a user model for life from the moment we were born and that model was updated constantly with our every day interactions with various online services? What if we had absolute control over it and we decided which system gets access to which part of our model? What if we could set the privacy status of our information? Then, we would be able to scrutinise it the way we wanted and benefit from any interactions we chose to make with any service out there, by providing our model before the interaction, and receiving it back at the end, updated with the new resulting data based on how we interacted with that service.

Consider the following imaginary scenario: Joe is a computer science student and for his programming assignment is required to develop a calculator written in Java. Joe knows only the basics around the Java programming language since he has read a book, bought from Amazon, and has gone through a couple of tutorials found on the course's website. In order to cope with the requirements of the assignment, Joe needs to register with personalization system XYZ and follow a short course on advanced concepts of Java. Joe logs on this new service, which we propose in this paper, and exports his Amazon model to XYZ, having previously set his Amazon model's privacy status to public. Furthermore, Joe filters his browsing history and selects to send to $\mathrm{XYZ}$ only the part that shows that he has gone through the Java tutorials. Now, XYZ knows what Joe is familiar with according to his various models and adapts its content accordingly to teach Joe Java concepts and features that he is unfamiliar with but are essential for the completion of his assignment.

What are the requirements for adopting a scrutable user modeling architecture and a communication protocol, for users AND providers of user models, from the educational, business and social networking domains, for enabling exchange of user models amongst them?

\section{Personalization \& User Modeling}

Personalization is essential for standalone and webbased systems aiming to offer effective and efficient services to their users. Every person must be considered independently and every application has to personalise its content according to their users' needs and characteristics.

User Modeling (UM) is the 'heart' of personalization services. By keeping a record, a model for each user, it allows unique adaptation and presentation of the available resources based on these models, thus enabling successful interactions between every user and available source.

Life-long UM was introduced in an attempt to model users under the new conditions, as were set by the new emerged Semantic Web. Consequently, new challenges such as: interoperable user profiles, scrutable UM solutions and user privacy components, have to be addressed and resolved.

\subsection{Interoperability}

Exchanging user profiles across various sources in a distributed eLearning (and not only) environments has become a flaming issue in UM. Explicit and widely accepted protocols have been introduced, which allow description, discovery and exchange of user models, 
stored in various systems - written in different languages and for different platforms $[2,3]$.

\subsection{Scrutability \& User Privacy}

The term scrutability in user modeling signifies that every user's model can be inspected and altered by its owner in order to determine what has been modelled about $\mathrm{him} / \mathrm{her}$ and how that modeling process was conducted [4].

The benefit of adopting srutability in UM can be indentified when users are allowed to select in which stereotypes they should be included and which ones they should not. Furthermore, scrutable solutions allow users to alter the value of any single inference that is used for drawing conclusions about them [4].

Privacy of user information is always a crucial issue in UM and privacy components should be included in every UM solution. Users should be given the option to set the status of their information and allowed to decide who can see which part of their user models.

\subsection{Semantic Web Technologies in User Modeling}

New directions and guidelines for UM have arisen with the introduction of the Semantic Web [1]. New technologies, such as XML, XML Schema, RDF, RDF Schema, OWL and Web Services, have emerged that allow the content of user models to be expressed in a format that can be read and processed by software agents, thus permitting them to find, share and integrate information more easily and efficiently.

\section{Proposed Solution}

A Scrutable User Modeling Infrastructure (SUMI) is presented which blends together these User Modeling "ingredients" and by taking advantage of Semantic Web technologies attempts to accomplish its primary goals, which are to:

- Achieve interoperability across the educational, business and social networking domains, while exchanging user models between SUMI and various user models' providers by adopting well accepted user modeling standards.

- Identify the possible extent for such architecture, to allow users to scrutinize their models and express their data privacy preferences.

- Collect the requirements of providers of user models for employing such a scrutable architecture.

A prototype has been designed and implemented. More specifically:
- Every model in SUMI is a representation of an integrated variety of models every user holds, by interacting with various services on the World Wide Web.

- Every user has absolute control over his/her SUMI model. The user can decide which models to integrate in SUMI and who gets to see which part of his/her SUMI model.

- Importing models from services can be achieved by adopting the SUMI import protocol, based on Semantic Web technologies.

- Every user is allowed to define the status of the data retrieved by his/her various models. The three categories of data are public, private and hidden.

- The most important feature of SUMI is the users' ability to export a part of their SUMI model to any registered service they prefer. This is achieved by adopting the SUMI export protocol, again based on Semantic Web technologies.

\section{Conclusion}

A Scrutable User Modeling Infrastructure has been introduced as a potential solution for enriching life-long User Modeling. Further research has already been identified which will target on gathering the requirements for adopting a scrutable user modeling architecture - and a communication protocol - that will allow exchange of user models to take place amongst web-based services from the educational, business and social networking domains.

\section{References}

[1] Berners-Lee, T., Hendler, J., Lassila, O., The Semantic Web. Scientific American, 284, 5 (2001) 34-43.

[2] Dolog, P., Schaefer, M., A Framework for Browsing, Manipulating and Maintaining Interoperable Learner Profiles. In Proc. of UM2005 - 10th International Conference on User Modelling, Edinburgh, UK, Springer-Verlag, (2005a).

[3] Dolog, P., Schaefer, M., Learner modelling on the semantic web. In Proc. of PerSWeb-2005 Workshop: Personalisation on the Semantic Web at User Modelling 2005: 10th International Conference, Edinburgh, UK, (2005b).

[4] Kay, J., Stereotypes, Student Models and Scrutability. In Proceedings of the 5th international Conference on intelligent Tutoring Systems, G. Gauthier, C. Frasson, and K. VanLehn, Eds. Lecture Notes in Computer Science, vol. 1839, London, Springer-Verlag, (2000) 19-30. 